\title{
A Gauge Theory of Nucleonic Interactions by Contact
}

\author{
Nicolae Mazilu \& Maricel Agop
}

\begin{abstract}
A gauge theory of contact is presented, based on the general idea that the local deformation of the nucleon surface at contact should be gauged by the variation of curvature. A contact force is then defined so as to cope with both the variation of curvature, and the deformation. This force generalizes the classical definition of surface tension, in that it depends on the mean curvature, but also depends on the variance of the second fundamental form of surface, considered as a statistical variable over the ensemble of contact spots. It turns out that the variance of the second fundamental form does not depend but on the metric of the space of curvature parameters, organized as Riemann space. This result compels us to review the definition of physical surface of a nucleon.
\end{abstract}

Key Words: curvature, curvature parameters, mean curvature, Gaussian curvature, contact force, $\mathfrak{s l}(2, \mathrm{R})$ Lie algebra, Cartan-Killing metric, gauge theory, gauging procedure.

\section{Introduction}

In a previous work (Mazilu \& Al., 2013) we corroborated some general results about the confining nuclear potential, pointing out to a way to take into consideration, unequivocally, the space expansion of the matter. This is a physically intuitive property of the matter, and should be quite critical, especially at the nuclear level. We have shown, for instance, that the logarithmic potential, so much circulated in numerical lattice calculations of the confining nuclear potential, can have sound explanations not just as a conjecture, but as a matter of principle, and hinted to the fact that this very principle should rest upon the property of color of matter in general. Even though at the theoretical level of elementary particles the color is considered no more than a quantum number, there are reasons to believe that this quantum number has a classical counterpart in the theory of light colors (Mazilu \& Al., 2014), and that, for instance, the principle of asymptotic freedom in the theory of strong and weak interactions is an expression of this very fact. Intuitively, the color is due to the space expansion of the matter, and is a phenomenon due to its confining. This phenomenon occurs at the limit between matter and space. As, classically speaking, such a limit is modeled by a surface, the present work focuses on a special issue involving the idea of surface in the nuclear realm: that of the contact of nucleons in a nucleus.

The concrete trend of our line of research is to start from results already available in theoretical fields where the space expansion of the bodies is essential. One such field, with exquisite theoretical results based on the differential geometry of surfaces, is the description of abrasion phenomenon as a dynamical process at the end of which the bodies of a certain congregation, pebbles for instance, acquire round shapes. This is plainly the case in geology, whence, as a matter of fact, the theory originated (Firey, 1974; Bloore, 1977). We were lucky enough to have found, already published, a fresh work with an up-to-date bibliography (Domokos, Gibbons, 2013), and eagerly recommend it for a general idea on the dynamics of the process of shape changing of extended bodies and the problems involved therein. This exquisite review is quite a comprehensive introduction in the problem, with the latest results, thus allowing us to shorten the discourse 
and focus on stressing out the essential points of our present effort which, while concentrating upon the same type of problems, takes another route: that of gauge-theoretical approach.

One can get a grip on the line of thought involved in this approach from a few works by Shapere and Wilczek, who treated this way the problem of swimming motion by shape change (Shapere, Wilczek, 1989a, $\mathbf{b}, \mathbf{c})$. The gauge field is defined here with reference to the rotation group acting on the space of shapes, and the tensor of force governing the dynamics is given by forces calculated from the variation of stress due to the change of shape. The time of the problem is obviously the classical time, and the treatment is, geometrically speaking, a'global' one, unlike the problem of wearing of pebbles, where one has to focus on local contacts, and consider a statistic of these, in order to evaluate the change in shape. The attractive general idea of gauge-theoretical approach is the fact that the surface changes of an extended body are the ones deciding the gauge field potentials. We adopt this idea here, but with no reference to an a priori time, and not from a global geometrical point of view, for it seems natural that in a conglomerate of particles - like the pebbles - the local contacts are essential, and the force should actually be defined at a contact.

Thus, the whole idea of this work is that the nucleus can be conceived indeed as a collective of 'pebbles'. Inside nucleus, these 'pebbles' - the nucleons, or congregations of nucleons - interact by contact, exactly like the real life pebbles, continuously adjusting each other's surface. In order to carry this analogy over completely into nuclear realm, two ideas have to be worked out in detail, both related to the notion of contact, however not quite independent from one another, at least from a physical point of view. First of all, because the nucleons, considered as extended particles, are exterior to one another, they interact through spots of their exterior surfaces, around the points of contact. Our view here is that of a general contact: it can be, in the last resort, even characterized by a 'proximity', in a manner occasionally done before for the theory of nucleus (Błocki, Świątecki, 1981), involving pressures. These can be conceived as being of different intensities, even negative, in which case the contact should be 'virtual', i.e. the surfaces are at distance with respect to one another along the line of contact. Our main problem is therefore to characterize such a contact in the most general way. Secondly, even though the nucleons are assimilated with pebbles, one should keep always in mind the fact that they are not quite like ordinary pebbles from a material characterization point of view. Therefore they have to be described in a general way, allowing for an appropriate constitutive law, essential, as we see it, in the calculations of the confining potential, as our previous work shows (Mazilu \& Al, 2013). This point will not be touched here though, but in a future communication.

Rather, we shall presently concentrate upon the general geometrical description of the contact, within the local description of surfaces. We start from the obvious observation that a contact is a local event, involving therefore a local deformation of the surfaces of nucleons, a process through which the physics gets in. A variant of the classical theory of surfaces, involving differential forms, then allows us to gradually introduce that physics into the process of deformation, through a special statistics. This is actually the whole task of the present theoretical effort.

The mathematical method itself, for carying out the task, is based on an almost trivial statement that emerged apparently largely unnoticed. In order to make it obvious, we reproduce here two of the Élie Cartan's algebraical theorems which form the ground of his approach of differential geometry by moving frames (for a clear description of the concept, from the point of view we adopt in the present work, see Spivak 1999, Vol. II, Chapter 7). These are adapted directly from Cartan's course, via the Russian geometrical school of S. P. Finikov (Cartan, 2001; pp. 16 - 17, Theorems 7 \& 9), and we call them here Cartan Lemmas 1 and 2 , only in order to be used in the present context:

Lemma 1. Suppose that $\mathrm{s}^{1}, \mathrm{~s}^{2}, \ldots, \mathrm{s}^{\mathrm{p}}$ is a set of linearly independent 1 -forms. Then we have

$$
\mathrm{s}^{\mathrm{k}} \wedge \phi_{\mathrm{k}}=0 \Leftrightarrow \phi_{\mathrm{k}}=\mathrm{a}_{\mathrm{kj}} \mathrm{s}^{\mathrm{j}} ; \mathrm{a}_{\mathrm{ij}}=\mathrm{a}_{\mathrm{ji}}
$$


where $\phi_{1}, \phi_{2}, \ldots, \phi_{\mathrm{p}}$ is another set of linearly independent 1 -forms, and summation over repeated indices is understood.

Lemma 2. Suppose the basis differential elements $\mathrm{du}^{1}, \mathrm{du}^{2}, \ldots, \mathrm{du}^{\mathrm{n}}$ are connected by a system of equations

$$
\omega^{1}=0, \quad \omega^{2}=0, \quad \ldots \quad \omega^{\mathrm{p}}=0
$$

where $\omega^{\mathrm{j}}, \mathrm{j}=1,2, \ldots, \mathrm{p}$ are linearly independent 1 -forms. Then the 2 -form $\mathrm{f}$ vanishes as a consequence of this system if, and only if, $\mathrm{f}$ can be written as

$$
\mathrm{f}=\omega^{\mathrm{k}} \wedge \phi_{\mathrm{k}}
$$

where, again, summation over $\mathrm{k}$ is understood, and $\phi_{\mathrm{k}}$ are $\mathrm{p}$ conveniently chosen 1-forms.

The first one of these theorems is, by and large, known as Cartan's Lemma proper in the specialty literature, while the second one carries no specific name. What seems to be essential in these lemmas, and is always stressed in old treatises, but is apparently forgotten lately, is the fact that symmetric matrix a from Lemma 1 and the 1 -forms $\phi_{\mathrm{k}}$ from Lemma 2 are things external to the geometrical problem, through which the physics can therefore be introduced. In concentrating on the local contact in a point of a surface, without being interested of the global aspects of that surface, this observation is essential.

\section{Classical Theory of Surfaces}

Cartan's differential form approach to the theory of surfaces (Guggenheimer, 1977), allows us to say that the local differential theory of surfaces is simply a consequence of the fact that the elementary (differential) displacement of a point of surface is an intrinsic vector of that surface. Everything in the differential theory of surfaces follows from this simple fact through the rules of handling of the differential forms. To wit, if $\vec{x}$ is the position vector of a point of surface, then $d \vec{x}$ should be an intrinsic vector of the surface, at least as long as no physical phenomenon occurs forcing the position to leave the surface. But even in such a case, instrumental from a physical point of view, the elementary variation of position can be described by a process of deformation through a continuous family of surfaces containing the position during that variation.

Let us now lay down the proper mathematical form of this portrayal of surfaces. Referring the local geometry at location $\vec{x}$ on the surface to an orthonormal frame $\left(\hat{\mathrm{e}}_{1}, \hat{\mathrm{e}}_{2}, \hat{\mathrm{e}}_{3}\right)$, where $\hat{\mathrm{e}}_{3}$ is the unit normal at that location, we write

$$
\mathrm{d} \overrightarrow{\mathrm{x}}=\mathrm{s}^{1} \hat{\mathrm{e}}_{1}+\mathrm{s}^{2} \hat{\mathrm{e}}_{2} \quad \leftrightarrow \mathrm{d} \overrightarrow{\mathrm{x}}=\mathbf{s}|\hat{\mathrm{e}}\rangle
$$

The second equality in this equation involves a "Dirac representation" of the vectors, to be used extensively here, quite handy in calculations, whereby, mostly for a fixed reference frame, the vectors are represented by matrices, regardless of the nature of their components and the number of dimensions of space. By the same token, the matrices themselves will be always represented by bold letters, no matter of case and array dimensions. For instance, in equation (1) we have:

$$
\mathbf{s} \equiv\left(\begin{array}{cc}
\mathrm{s}^{1} & 0 \\
0 & \mathrm{~s}^{2}
\end{array}\right), \quad|\hat{\mathrm{e}}\rangle \equiv\left(\begin{array}{c}
\hat{\mathrm{e}}_{1} \\
\hat{\mathrm{e}}_{2}
\end{array}\right), \quad\langle\hat{\mathrm{e}}|=| \hat{\mathrm{e}}\rangle^{\mathrm{t}} \equiv\left(\begin{array}{ll}
\hat{\mathrm{e}}_{1} & \hat{\mathrm{e}}_{2}
\end{array}\right),
$$

and the same notations will be used for the vectors in space, if the case may occur. This notation for vectors will be used freely in conjunction with the general notation by an arrow over the letter designating the vector, mostly when the reference frame itself is meant to vary.

Now, according to differential calculus, the vector $\mathrm{d} \overrightarrow{\mathrm{x}}$ is a total differential vector, so that its exterior differential is the null vector. This can be expressed by equation

$$
\mathrm{d} \wedge \mathrm{d} \overrightarrow{\mathrm{x}}=\overrightarrow{0} \quad \therefore \quad(\mathrm{d} \wedge \mathbf{s})|\hat{\mathrm{e}}\rangle+\langle\mathrm{d} \hat{\mathrm{e}}| \wedge \mathbf{s}=\overrightarrow{0}
$$


where " $\wedge$ " means "exterior" for the designated operation (differentiation or multiplication). As to the variation of the reference frame, it is usually described by a Frenet-Serret evolution equation:

$$
|\mathrm{dê}\rangle=\mathbf{\Omega} \cdot|\hat{\mathrm{e}}\rangle
$$

where $\boldsymbol{\Omega}$ is a skewsymmetric $3 \times 3$ matrix. In detail, the equation (4) appears as a system:

$$
d \hat{\mathrm{e}}_{1}=\omega_{1}^{2} \hat{\mathrm{e}}_{2}+\omega_{1}^{3} \hat{\mathrm{e}}_{3}, \quad \mathrm{~d} \hat{\mathrm{e}}_{2}=\omega_{2}^{1} \hat{\mathrm{e}}_{1}+\omega_{2}^{3} \hat{\mathrm{e}}_{3}, \quad \mathrm{~d} \hat{\mathrm{e}}_{3}=\omega_{3}^{1} \hat{\mathrm{e}}_{1}+\omega_{3}^{2} \hat{\mathrm{e}}_{2}
$$

The last equation here represents the fact that the variation of the unit normal to surface is an intrinsic vector. As known, this vector expresses the local curvatures of the surface at the chosen location, which are obtained by its projections in different directions of the surface. So, for our specific needs it will be designated from now on as the curvature vector. With the help of system (5), the second equality from equation (3) becomes itself a system:

$$
\mathrm{d} \wedge \mathrm{s}^{1}+\omega_{2}^{1} \wedge \mathrm{s}^{2}=0, \quad \mathrm{~d} \wedge \mathrm{s}^{2}+\omega_{1}^{2} \wedge \mathrm{s}^{1}=0, \quad 0=\omega_{1}^{3} \wedge \mathrm{s}^{1}+\omega_{2}^{3} \wedge \mathrm{s}^{2}
$$

The first two equations of this system express the fact that, in general, the components of the elementary displacement on the surface may not be exact differentials, but they can be always taken as exact differentials in cases where the connection form vanishes (a trivial instance is the case of a plane surface). The last equation of the system (6) can be exploited with the help of the Cartan's Lemma 1, thus leading to a definition of the curvature matrix, which we represent conveniently by

$$
\left(\begin{array}{l}
\omega_{1}^{3} \\
\omega_{2}^{3}
\end{array}\right)=-\left(\begin{array}{ll}
\alpha & \beta \\
\beta & \gamma
\end{array}\right) \cdot\left(\begin{array}{l}
s^{1} \\
s^{2}
\end{array}\right) \leftrightarrow\left|\omega^{3}\right\rangle=-\alpha \cdot|s\rangle
$$

with $\alpha, \beta, \gamma$ some parameters - the curvature parameters.

The trace of the curvature matrix gives the mean curvature, and its discriminant gives the Gaussian curvature. Our contention here, inspired by this way of introducing the curvature parameters, is that these are actually external parameters, possibly physical, a feature bestowed upon them by the Cartan's lemma. Fact is that they are always subjected to variations when physical causes, like the contact of bodies, participate in changing the local aspect of their surface. However, their evaluation - in case one needs it, and has the conditions to do it, of course - can be done just by geometrical measurements on the surface, as in the usual classical procedure (Lowe, 1980).

The sign in equation (4) is so chosen that the projection of the second variation of the position vector along the normal to surface in the given point, is a quadratic form without sign. Thus:

$$
\mathrm{d} \overrightarrow{\mathrm{x}} \cdot \hat{\mathrm{e}}_{3}=0 \Rightarrow \mathrm{d}^{2} \overrightarrow{\mathrm{x}} \cdot \hat{\mathrm{e}}_{3}=-\mathrm{d} \overrightarrow{\mathrm{x}} \cdot \mathrm{d} \hat{\mathrm{e}}_{3}=\langle\mathrm{s}|\boldsymbol{\alpha}| \mathrm{s}\rangle
$$

because of the skew symmetry of the matrix $\boldsymbol{\Omega}$. Obviously, the quadratic form from the right hand side here is the second fundamental form of the surface at the given location. Thus, one can say that, in the tangent plane of a surface at a given point, every vector is a linear combination between the elementary displacement and the curvature vector:

$$
|\mathrm{V}\rangle=\lambda|\mathrm{s}\rangle+\mu\left|\omega^{3}\right\rangle \leftrightarrow|\mathrm{V}\rangle=(\lambda \mathbf{e}-\mu \boldsymbol{\alpha}) \cdot|\mathrm{s}\rangle
$$

where $\mathbf{e}$ is the identity $2 \times 2$ matrix, and $\lambda, \mu$ are two real parameters. The norm of such a vector is a quadratic form in the parameters $\lambda$ and $\mu$ :

$$
\langle\mathrm{V} \mid \mathrm{V}\rangle=\lambda^{2}\langle\mathrm{~s} \mid \mathrm{s}\rangle+2 \lambda \mu\left\langle\mathrm{s} \mid \omega^{3}\right\rangle+\mu^{2}\left\langle\omega^{3} \mid \omega^{3}\right\rangle
$$

having as coefficients the three fundamental forms of the surface at the chosen location:

$$
\begin{aligned}
\langle\mathrm{V} \mid \mathrm{V}\rangle & =\lambda^{2}\langle\mathrm{~s} \mid \mathrm{s}\rangle-2 \lambda \mu\langle\mathrm{s}|\boldsymbol{\alpha}| \mathrm{s}\rangle+\mu^{2}\left\langle\mathrm{~s}\left|\boldsymbol{\alpha}^{2}\right| \mathrm{s}\right\rangle \\
& =\lambda^{2} \mathrm{I}-2 \lambda \mu \mathrm{II}+\mu^{2} \mathrm{III}
\end{aligned}
$$

The three bracketed coefficients of this quadratic form are, indeed, the fundamental forms of the surface at the chosen location: the first (I), the second(II) and the third (III). 


\section{Nonconstant Curvature}

We apply a Cartan gauging procedure (Pommaret, 2013) at a generic - but fixed - point from the tangent plane at a given position on the surface, infinitesimally close to that position. Because our point is fixed, we have from equation (7), in the Dirac notation:

$$
\left|\mathrm{d} \omega_{3}\right\rangle=\mathrm{d} \boldsymbol{\alpha}|\mathrm{s}\rangle
$$

as an equation for the 'orbits' through $|s\rangle$, as it were. Now, using equation (7) itself, as the one determining those orbits, the equation (8) gives actually a definition of the variation of curvature vector in a certain point of the tangent plane, infinitesimally close to the point of contact:

$$
\left|\mathrm{d} \omega_{3}\right\rangle=\left(\mathrm{d} \boldsymbol{\alpha} \cdot \boldsymbol{\alpha}^{-1}\right)\left|\omega_{3}\right\rangle
$$

Each and every point $|s\rangle$ from a tangent plane, at a given position on the surface, is characterized by such an equation defining the variation of curvature vector. Using the curvature parameters from equation (7) explicitly, the equation (9) is:

$$
\left|\mathrm{d} \omega_{3}\right\rangle=(\mathrm{d} \lambda \mathbf{e}+\boldsymbol{\omega})\left|\omega_{3}\right\rangle
$$

where we denoted:

$$
\lambda=\ln \sqrt{\alpha \gamma-\beta^{2}} ; \quad \boldsymbol{\omega}=\left(\begin{array}{cc}
-\omega_{2} / 2 & \omega_{1} \\
-\omega_{3} & \omega_{2} / 2
\end{array}\right)
$$

with $\omega_{1,2,3}$ the differential 1-forms:

$$
\omega_{1}=\frac{\alpha \mathrm{d} \beta-\beta \mathrm{d} \alpha}{\alpha \gamma-\beta^{2}} ; \quad \omega_{2}=\frac{\alpha \mathrm{d} \gamma-\gamma \mathrm{d} \alpha}{\alpha \gamma-\beta^{2}} ; \quad \omega_{3}=\frac{\beta \mathrm{d} \gamma-\gamma \mathrm{d} \beta}{\alpha \gamma-\beta^{2}}
$$

Now, in order to interpret geometrically, and therefore physically, this definition, we need first to have some characterization of the vector from equation (10) with respect to the position $|s\rangle$ from the tangent plane. This will then show how much the second fundamental form differs from the nominal one, calculated in the origin of the plane. From equation (10) we have by direct dot multiplication:

$$
\left\langle\mathrm{s} \mid \mathrm{d} \omega_{3}\right\rangle=(\mathrm{d} \lambda)\left\langle\mathrm{s} \mid \omega_{3}\right\rangle+\left\langle\mathrm{s}|\omega| \omega_{3}\right\rangle
$$

Therefore the curvature variation 'gauged' through equation (10) induces a differential variation along the position vector in the tangent plane, proportional with the second fundamental form, but to which a certain quadratic form is added. Using equation (7) for calculating this quadratic form, results in

$$
\left\langle\mathrm{s}|\boldsymbol{\omega}| \omega_{3}\right\rangle \equiv\langle\mathrm{s}|\boldsymbol{\omega} \cdot \boldsymbol{\alpha}| \mathrm{x}\rangle=\left(\beta \omega_{1}-\frac{\alpha}{2} \omega_{2}\right)\left(\mathrm{s}^{1}\right)^{2}+\left(\gamma \omega_{1}-\alpha \omega_{3}\right) \mathrm{s}^{1} \mathrm{~s}^{2}+\left(\frac{\gamma}{2} \omega_{2}-\beta \omega_{3}\right)\left(\mathrm{s}^{2}\right)^{2}
$$

The general conclusion is that the variation of curvature parameters at a given position on the surface, involves three quadratic differential forms: the second fundamental form of surface in the given position, the quadratic differential from equation (14) and the quadratic differential

$$
-\left\langle\left.\mathrm{d} \omega_{3}\right|^{*} \mathrm{~s}\right\rangle=\omega_{1}\left(\mathrm{~s}^{1}\right)^{2}+\omega_{2} \mathrm{~s}^{1} \mathrm{~s}^{2}+\omega_{3}\left(\mathrm{~s}^{2}\right)^{2}
$$

where the star denotes the usual Hodge duality operation on the differentials. Now, the two differential forms involved in equation (13), together with the one from equation (15) are, from algebraic point of view, a system of three mutually apolar quadratics (for the explanation of this concept see Burnside, Panton, 1960, pp. 333-334). The idea is thereby suggested, that the apolarity plays a fundamental role, when it comes to issues of the variation of curvature, and consequently to the physics involved in this variation of curvature.

Just for the benefit of present discussion, the algebraic apolarity is understood here as follows: two quadratics written in the form

$$
a_{1} x^{2}+2 b_{1} x y+c_{1} y^{2}, \quad a_{2} x^{2}+2 b_{2} x y+c_{2} y^{2}
$$

are apolar if their covariant 


$$
a_{1} c_{2}-2 b_{1} b_{2}+a_{2} c_{1}
$$

vanishes. The meaning of this fact is that the two corresponding quadratic equations (considered in a nonhomogeneous variable $\mathrm{x} / \mathrm{y}$, of course) have roots in a harmonic range: the cross-ratio of these roots, taken in a certain order, is -1 . This is why two such quadratics are sometimes designated as mutually harmonic, mostly in the old classical treatises of algebra (see Burnside, Panton, 1960).

The whole physics involved in the local variation of curvature in a given position of a certain surface is therefore contained in the following reading of the previous calculus: an external agent somehow defines infinitesimal variations of the curvature parameters, which can be gauged by equation (10). These variations change the second fundamental form of the surface which, according to equation (13), becomes

$$
\left\langle\mathrm{s} \mid \omega_{3}+\mathrm{d} \omega_{3}\right\rangle=(1+\mathrm{d} \lambda)\left\langle\mathrm{s} \mid \omega_{3}\right\rangle+\langle\mathrm{s}|\boldsymbol{\omega} \cdot \boldsymbol{\alpha}| \mathrm{s}\rangle
$$

The two quadratic forms entering this expression of the new second fundamental form are apolar with the quadratic form (15) representing, as we shall see, some variation of the second fundamental form.

The physics can even be introduced in general terms, suggested by the generality of Cartan's Lemma 1. Indeed, if the external perturbation is represented by a quadratic form with coefficients a, b, c say, viz.

$$
\mathrm{U}(\mathrm{s})=\mathrm{a}\left(\mathrm{s}^{1}\right)^{2}+2 \mathrm{bs} \mathrm{s}^{1} \mathrm{~s}^{2}+\mathrm{c}\left(\mathrm{s}^{2}\right)^{2}
$$

then it changes the second fundamental form of the surface according to equation

$$
\mathrm{II}^{\prime}=[1+\Delta(\mathrm{U})] \cdot \mathrm{II}+(\beta \mathrm{a}-\alpha \mathrm{b})\left(\mathrm{s}^{1}\right)^{2}+(\gamma \mathrm{a}-\alpha \mathrm{c}) \mathrm{s}^{1} \mathrm{~s}^{2}+(\gamma \mathrm{b}-\beta \mathrm{c})\left(\mathrm{s}^{2}\right)^{2}
$$

where $\Delta(\mathrm{U})$ is a parameter depending only on the coefficients of $\mathrm{U}(\mathrm{s})$.

\section{The Infinitesimal Deformation}

In order to give an interpretation of the previous results we call upon one of the many description of the deformation of surfaces, namely the infinitesimal deformation (Guggenheimer, 1977, p. 245ff). Such a deformation gives a new surface described by the position vector

$$
\overrightarrow{\mathrm{r}}(\varepsilon)=\overrightarrow{\mathrm{x}}+\varepsilon \cdot \overrightarrow{\mathrm{z}}
$$

By its definition, this type of deformation is such that the variation of the first fundamental form is null for small $\varepsilon$, i.e. it remains approximately constant through the deformation:

$$
\lim _{\varepsilon \rightarrow 0} \frac{I_{\vec{r}}-I_{\vec{x}}}{\varepsilon}=0
$$

This condition leads to the following constraint for the vector $\vec{z}$ :

$$
\mathrm{d} \overrightarrow{\mathrm{x}} \cdot \mathrm{d} \overrightarrow{\mathrm{z}}=0 \quad \therefore \mathrm{d} \overrightarrow{\mathrm{z}}=\overrightarrow{\mathrm{y}} \times \mathrm{d} \overrightarrow{\mathrm{x}}
$$

$\vec{y}$ here is an auxiliary vector, which cannot be quite arbitrary. Its properties are again simple consequences of the exterior differential calculus. Indeed, the vector $\mathrm{d} \overrightarrow{\mathrm{z}}$ in equation (21) should be an exact differential vector. Imposing this condition on the second of equations (21), and using again Cartan's Lemma 1, leads directly to the following equations, where the definition $\vec{v} \equiv \mathrm{d} \vec{y}$ is used:

$$
\mathrm{v}^{1}=\mathrm{Bs}^{1}+\mathrm{Cs}^{2}, \quad-\mathrm{v}^{2}=\mathrm{As}^{1}+\mathrm{Bs}^{2}, \quad \mathrm{v}^{3}=0
$$

Thus, the vector $|\mathrm{v}\rangle$ as defined here is practically an intrinsic vector of surface, which means that the normal component of the vector $|y\rangle$ itself must be, locally, a constant. Moreover, by its definition $\vec{v}$ must be an exact differential vector. The condition that its exterior differential is the null vector, leads to equations like (6), but for $\mathrm{v}^{1}$ and $\mathrm{v}^{2}$. The third of those, combined with equation (22), then shows that A, B, C are constrained to satisfy the linear relation

$$
\alpha \mathrm{C}+\gamma \mathrm{A}-2 \beta \mathrm{B}=0
$$

where $\alpha, \beta, \gamma$ are the coefficients of the second fundamental form (the entries of the curvature matrix). This shows that the flux through the tangent plane of the surface at the chosen location, is given by equation: 


$$
\mathrm{d} \overrightarrow{\mathrm{x}} \times \overrightarrow{\mathrm{v}}=\left\{\mathrm{A}\left(\mathrm{s}^{1}\right)^{2}+2 \mathrm{~B} \mathrm{~s}^{1} \mathrm{~s}^{2}+\mathrm{C}\left(\mathrm{s}^{2}\right)^{2}\right\} \cdot \hat{\mathrm{e}}_{3}
$$

has as magnitude a quadratic form, which is apolar to the second fundamental form. It only remains to elaborate on how the coefficients A, B, C can be related to the physics of our problem.

\section{Infinitesimal Deformation Induced by Variation of Curvature Parameters}

The parameters A, B, C are, again, external parameters, introduced by Cartan's Lemma 1. We have noticed that the equation (23) is an apolarity condition, defining the apolarity between the quadratic form (24) and the second fundamental form in the point of surface considered. If we take these parameters as differential forms according to the rule of correspondence

$$
\mathrm{A} \leftrightarrow \omega_{1} ; \quad 2 \mathrm{~B} \leftrightarrow \omega_{2} ; \quad \mathrm{C} \leftrightarrow \omega_{3},
$$

we can define an infinitesimal deformation, induced exclusively by the variation of curvature parameters. This infinitesimal deformation is then expressed by an addition to the second fundamental form, as we already mentioned before, given by equation (24) as:

$$
-\vec{v} \times d \vec{x}=\left\{\omega_{1}\left(s^{1}\right)^{2}+\omega_{2} s^{1} s^{2}+\omega_{3}\left(s^{2}\right)^{2}\right\} \cdot \hat{e}_{3}
$$

This vector has indeed the magnitude given by equation (15), and therefore can be viewed as related somehow to the vector $\left|\mathrm{d} \omega^{3}\right\rangle$ defined in equation (9). It can be described by an auxiliary vector $|\mathrm{y}\rangle$ defining the deformation, which is directed along the normal to surface, and which acquires in-surface components by the very curvature parameters' variations:

$$
\left(\begin{array}{l}
0 \\
0 \\
y^{3}
\end{array}\right) \rightarrow\left(\begin{array}{c}
\left(\omega_{2} / 2\right) s^{1}+\omega_{3} s^{2} \\
-\omega_{1} s^{1}-\left(\omega_{2} / 2\right) s^{2} \\
y^{3}
\end{array}\right)
$$

The corresponding first fundamental form on the deformed surface then becomes:

$$
\mathrm{I}=\langle\mathrm{s}|\mathbf{h}| \mathrm{s}\rangle, \quad \mathbf{h}=\left\{1+\left(\varepsilon \mathrm{y}^{3}\right)^{2}\right\} \mathbf{e}+\varepsilon^{2}(\boldsymbol{\varepsilon} \cdot|\mathrm{v}\rangle\langle\mathrm{v}| \cdot \boldsymbol{\varepsilon})
$$

where $|\mathrm{v}\rangle$ is defined in equation (22) but with the values (25) for the coefficients $\mathrm{A}, \mathrm{B}, \mathrm{C}$. This matrix obviously reduces to the usual identity matrix, once the parameter $\varepsilon \rightarrow 0$.

\section{Summing Up the Differential Geometry of Curvature Parameters}

A few algebraical relations among the differential forms from equation (12) are in order. They form a basis (coframe) of a $\mathfrak{s l}(2, \mathrm{R})$ algebra. We already alluded to a Lie group structure, by presenting equation (9) as a gauge equation referring to an 'orbit', obtained by left multiplication with the inverse of the curvature matrix. More than this, it turns out that, among other things, the space of curvature parameters can be organized as a Riemannian space. In order to show this, notice first the following differential relations in the space of curvature parameters, which can be proved by a direct calculation:

$$
\mathrm{d} \wedge \omega_{1}=\frac{\alpha}{\sqrt{\Delta}} \Theta ; \quad \mathrm{d} \wedge \omega_{2}=\frac{2 \beta}{\sqrt{\Delta}} \Theta ; \quad \mathrm{d} \wedge \omega_{3}=\frac{\gamma}{\sqrt{\Delta}} \Theta
$$

Here $\Theta$ is the differential 2-form

$$
\Theta \equiv \frac{\alpha \mathrm{d} \beta \wedge \mathrm{d} \gamma+\beta \mathrm{d} \gamma \wedge \mathrm{d} \alpha+\gamma \mathrm{d} \alpha \wedge \mathrm{d} \beta}{\Delta^{3 / 2}}
$$

The 2-form $\Theta$ is closed because it is the exterior differential of a 1-form:

$$
\Theta \equiv \mathrm{d} \wedge \psi ; \quad \psi \equiv \frac{\alpha+\gamma}{\sqrt{\Delta}} \mathrm{d}\left(\tan ^{-1} \frac{2 \beta}{\alpha-\gamma}\right)
$$

representing the classical Hannay angle for this problem. In the present context it can be written as 


$$
\psi=2 \frac{\left(\omega_{1}+\omega_{3}\right)}{\sinh \xi}, \quad \xi \equiv \ln \frac{\alpha+\gamma}{2 \sqrt{\Delta}}
$$

including explicitly the ratio between the mean and Gaussian curvatures. It gives therefore a way to found the mathematical procedure for the local contact problem, but certainly has everything in common with the original angle designated as such (Hannay, 1985; Berry, 1985).

On the other hand, we can verify the following relations:

$$
\omega_{1} \wedge \omega_{2}=\frac{\alpha}{\sqrt{\Delta}} \Theta ; \quad \omega_{2} \wedge \omega_{3}=\frac{\gamma}{\sqrt{\Delta}} \Theta ; \quad \omega_{3} \wedge \omega_{1}=-\frac{\beta}{\sqrt{\Delta}} \Theta
$$

Thus, from (29) and (32) we have indeed the characteristic equations of a $\mathfrak{s l}(2, \mathrm{R})$ structure:

$$
\mathrm{d} \wedge \omega_{1}-\omega_{1} \wedge \omega_{2}=0 ; \quad \mathrm{d} \wedge \omega_{3}-\omega_{2} \wedge \omega_{3}=0 ; \quad \mathrm{d} \wedge \omega_{2}+2\left(\omega_{3} \wedge \omega_{1}\right)=0
$$

Using these relations we can draw an important conclusion, destined to guide our future research. Notice, indeed, that in a given point of the surface, we can construct the 2-form in the curvature parameters' space:

$$
\langle\mathrm{s}|\mathrm{d} \wedge(\boldsymbol{\omega} \cdot \boldsymbol{\varepsilon})| \mathrm{s}\rangle=\langle\mathrm{s}|\boldsymbol{\alpha}| \mathrm{s}\rangle \frac{\Theta}{\sqrt{\Delta}} ; \quad \boldsymbol{\varepsilon} \equiv\left(\begin{array}{cc}
0 & -1 \\
1 & 0
\end{array}\right)
$$

where we have used the equation (29) and the matrix $\omega$ from equation (11). As the 2 -form $\Theta$ is a flux in the space of curvature parameters, viz. something analogous of the solid angle in the usual Euclidean space, the second fundamental form $\langle\mathrm{s}|\boldsymbol{\alpha}| \mathrm{s}\rangle$ of a surface, in a given point, is in fact the intensity (the value) of a flux in the space of curvature parameters, depending quadratically on the position in the local tangent plane. This philosophy can be used profitably, in constructing a statistic of the contact forces for a a nucleon in the nucleus. First, however, let us define the notion of contact force, in order to reveal the right place of the mark of Riemannian character of the space of curvature parameters: its metric.

\section{A Definition of Contact Force}

We introduce the contact force as a differential 2-form. It can be the component of a vector, or even the magnitude of a vector depending on the measure of the deformation of surface we manage to define. For, it is obvious that a local contact induces a local deformation of the surface, and that the force should be somehow related to this deformation. We define it as a 2-form:

$$
\mathrm{f}=\mathrm{d} \omega_{1}^{3} \wedge \phi^{1}+\mathrm{d} \omega_{2}^{3} \wedge \phi^{2}
$$

where $\phi^{1}$ and $\phi^{2}$ are two conveniently chosen differential 1-forms, and the components of the differential of curvature vector are defined in equation (10). The equation (35) incorporates a specific logic, allowed by Cartan's Lemma 2. According to this lemma, the force is zero whenever there is not a variation of curvature in the given point, and reciprocally: if the force is zero, there is no variation of curvature. This last fact is intuitively obvious for an idea of contact force. However, in calculating such a force, one obviously has to take in consideration some particular physical circumstances, embodied in the choice of differential 1-forms $\phi^{1}$ and $\phi^{2}$. The force can be zero even if there are curvature variations.

Thus, if the conveniently chosen auxiliary forms, in the definition (35) of the contact force, are the components of the first fundamental form, i.e. the components of the position vector $|s\rangle$ in the tangent plane, then equation (35) simply shows that there is no contact force. Indeed, using equation (10) in (35), with $\mathrm{s}^{1}$ and $\mathrm{s}^{2}$ instead of $\phi^{1}$ and $\phi^{2}$, this contact force should be

$$
\mathrm{f}=\left(\alpha \omega_{3}+\gamma \omega_{1}-\beta \omega_{2}\right)\left(\mathrm{s}^{1} \wedge \mathrm{s}^{2}\right)
$$

This is zero by the apolarity between the second fundamental form and the magnitude of vector from equation (26). Therefore, the whole point of this definition of the contact force is that it is simply zero for no deformation, but depends explicitly on the variations of the curvature parameters. 
Our example of infinitesimal deformation helps in further elucidating the idea. Assume, indeed, that a surface is deformed and this deformation can be expressed by a vector equation like equation (19). Now, if in equation (35) we choose the differential 1 -forms $\phi^{1,2}$ as the components of the displacement on the deformed surface, rather than the ones on undeformed surface as above, i.e.:

$$
\phi^{1}=\mathrm{s}^{1}+\varepsilon \mathrm{dz}^{1}, \quad \phi^{2}=\mathrm{s}^{2}+\varepsilon \mathrm{dz} \mathrm{z}^{2}
$$

then according to equation (35), the contact force is given by:

$$
\mathrm{f}=\varepsilon \mathrm{y}^{3}\left(-\mathrm{d} \omega_{1}^{3} \wedge \mathrm{s}^{2}+\mathrm{d} \omega_{2}^{3} \wedge \mathrm{s}^{1}\right)
$$

because by equation (21) for $|\mathrm{dz}\rangle$, we have

$$
\mathrm{dz}^{1}=-\mathrm{y}^{3} \mathrm{~s}^{2}, \quad \mathrm{dz} \mathrm{z}^{2}=\mathrm{y}^{3} \mathrm{~s}^{1}
$$

with $\mathrm{y}^{3}$ the constant normal component of the auxiliary vector $|y\rangle$. With (10), equation (37) then gets the form

$$
\mathrm{f}=\left(\varepsilon \mathrm{y}^{3}\right)\left\{(\alpha+\gamma) \mathrm{d} \lambda+\beta\left(\omega_{1}-\omega_{3}\right)+\frac{\omega_{2}}{2}(\gamma-\alpha)\right\}\left(\mathrm{s}^{1} \wedge \mathrm{s}^{2}\right)
$$

or, after a few calculations

$$
\mathrm{f}=\left(\varepsilon \mathrm{y}^{3}\right) \mathrm{d}(\alpha+\gamma)\left(\mathrm{s}^{1} \wedge \mathrm{s}^{2}\right)
$$

This is the classical form of the surface tension: the contact force is proportional with the variation of the mean curvature. It allows us to say something about the auxiliary vector $|y\rangle$, and then improve upon our definition of contact force.

First, we have to recall that the surface tension has always been judged, in the case of liquids for instance, by an equilibrium between the flux of internal molecular forces of a liquid, and the external global force, like the gravitation in the case of capillary phenomena. Hence the quantity $\varepsilon y^{3} d(\alpha+\gamma)$ in equation (38) should reflect such an equilibrium. One can say that the vector $|y\rangle$ is always related to an equilibrium of forces, even in the case of the most general contact. More than that, the small quantity $\varepsilon$ reflecting the magnitude of deformation should also be related to the equilibrium of forces over a contact spot.

\section{The Statistics of Contacts on a Particle}

The characterization of a local contact is closely related to a plane centric affine geometry. That is to say that if one insists in conducting a statistics of the contact forces on the surface of a certain nucleon, which is the natural course of thought in describing an internal dynamics of the nucleus, one has to consider the centric affine geometry in the tangent plane. This section shows a way to build such a statistics, based on the idea of continous Lie group characterizing the plane centric affine geometry.

The $\mathfrak{s l}(2, \mathrm{R})$ action preserving origin of this plane geometry is given by the three vectors

$$
X_{1}=s^{2} \frac{\partial}{\partial s^{1}} ; \quad X_{2}=\frac{1}{2}\left(s^{1} \frac{\partial}{\partial s^{1}}-s^{2} \frac{\partial}{\partial s^{2}}\right) ; \quad X_{3}=-s^{1} \frac{\partial}{\partial s^{2}}
$$

while the corresponding action in the space of curvature parameters is realized by the vectors:

$$
\mathrm{A}_{1}=-\alpha \frac{\partial}{\partial \beta}-2 \beta \frac{\partial}{\partial \gamma} ; \quad \mathrm{A}_{2}=-\alpha \frac{\partial}{\partial \alpha}+\gamma \frac{\partial}{\partial \gamma} ; \quad \mathrm{A}_{3}=2 \beta \frac{\partial}{\partial \alpha}+\gamma \frac{\partial}{\partial \beta}
$$

This last realization characterizes an intransitive action in the space of curvature parameters, which allows transitivity only along specific manifolds, given by constant discriminant of the second fundamental form. Therefore the action realized by operators (40) is transitive only at constant Gaussian curvature.

The functions of physical interest can be presented here as a joint invariants of any two of the actions given by equations (39) and (40), with the help of Stoka theorem (Stoka, 1968). According to this theorem, any joint invariant of the two actions is an arbitrary continuous function of the two algebraic formations 


$$
\alpha\left(s^{1}\right)^{2}+2 \beta s^{1} s^{2}+\gamma\left(s^{2}\right)^{2}, \quad \alpha \gamma-\beta^{2}
$$

Obviously the Gaussian probability density, for instance, if the case may occur, is only a special case of this theorem. By the same token, the straight lines through origin $s^{1}=s^{2}=0$ can be presented as joint invariants of two actions realized by operators (39), while the joint invariants of two actions realized by operators (40), one in the variables $\mathrm{a}, \mathrm{b}, \mathrm{c}$, say, the other in the curvature parameters $\alpha, \beta, \gamma$, are arbitrary continuous functions of the following three algebraic formations (Mazilu, 2004):

$$
\alpha \gamma-\beta^{2}, a c-b^{2}, a \gamma+c \alpha-2 b \beta
$$

These are important in problems transcending the manifolds of transitivity, like for instance transitions between points of different Gaussian curvatures from the surface of a nucleon, of which an example will be given presently. These algebraic facts can give good reasons for a few further observations related to the classical statistical theory of contact points at the surface of a nucleon inside nucleus.

Before entering the calculational detail, let us notice that such a line of thought hints toward ammending the definition of a shape as given by Shapere and Wilczek (loc. cit.). Namely, we consider the instant shape of a nucleon first of all as a collection of elementary events, described by contact points, their extended contact spots and the contact forces on them. It is this collection that should be considered as an evolving part of a 'phase space' of shapes. The actual space shapes have yet to be constructed from these elements by a certain mathematical procedure corresponding to physical facts. The classical illuminating example is Fresnel's construction of the wave surface from pieces accessible to diffraction experiments.

Thus, for instance, consider that the contact spots of a certain nucleonic surface, are dominated by the Dupin indicatrix at the contact points. According to Stoka theorem, the statistical ensemble of these contacts can be characterized by a normal probability density

$$
p_{X Y}\left(s^{1}, s^{2} \mid \alpha, \beta, \gamma\right) \equiv \frac{\sqrt{\alpha \gamma-\beta^{2}}}{2 \pi} \exp \left\{-\frac{1}{2}\left(\alpha\left(s^{1}\right)^{2}+2 \beta s^{1} s^{2}+\gamma\left(s^{2}\right)^{2}\right)\right\}
$$

in two statistical variables $\mathrm{X}$ and $\mathrm{Y}$, of which we don't know too much for now, other than that they are the coordinates of position on any one of the contact spots of the surface of nucleon, as suggested before.

We have, therefore, a way to calculate the statistics of a quadratic variable $\mathrm{Z}(\mathrm{X}, \mathrm{Y})$, obtained as before, in the process of deformation by contact, and having the generic values:

$$
\mathrm{z}\left(\mathrm{s}^{1}, \mathrm{~s}^{2}\right) \equiv \frac{1}{2}\left\{\mathrm{a}\left(\mathrm{s}^{1}\right)^{2}+2 \mathrm{bs}^{1} \mathrm{~s}^{2}+\mathrm{c}\left(\mathrm{s}^{2}\right)^{2}\right\}
$$

Thus we need to find first the probability density of this variable, under condition that the generic plane of contact is characterized by the a priori probability density as given, for instance, by the Gaussian in equation (43). The probability density of $Z$ should also satisfy the Stoka theorem, in the precise sense that it must be a function of the algebraic formations from equation (42). This leaves us with a functionally undetermined probability density though, even if we impose some natural constraints in order to construct it.

Nevertheless, proceeding directly, in the usual manner of the statistical theoretical practice, we are able to solve the problem, at least in this particular case, with important results. Thus, we have to find first the characteristic function of the variable (44). This is the expectation of the imaginary exponential of $\mathrm{Z}$, using (43) as probability density. Performing this operation directly, we get, with an obvious notation for the average:

$$
\left\langle\mathrm{e}^{\mathrm{i} \zeta \mathrm{Z}}\right\rangle=\frac{1}{2 \pi \sqrt{1+(\mathrm{i} \zeta) \frac{\mathrm{a} \gamma+\mathrm{c} \alpha-2 \mathrm{~b} \beta}{\mathrm{ac}-\mathrm{b}^{2}}+(\mathrm{i} \zeta)^{2} \frac{\alpha \gamma-\beta^{2}}{a c-b^{2}}}}
$$


In view of (42), this characteristic function certainly satisfies the Stoka theorem, which thus reveals its right place in a physical theory: it should serve for the selection of the right physical functions, specifically the probability density, or the characteristic function, as in this case. Anyway, the sought for probability density can then be found by a routine Fourier inversion of the characteristic function from equation (45), based on existing tabulated formulas (see Gradshteyn, Ryzhik, 1994; 2007, especially the examples 3.384(43); 6.611 (40); 9.215(16)\&(39)). The result is:

$$
\mathrm{p}_{\mathrm{z}}(\mathrm{z} \mid \alpha, \beta, \gamma)=\sqrt{\mathrm{AB}} \exp \left(-\frac{\mathrm{A}+\mathrm{B}}{2} \mathrm{z}\right) \cdot \mathrm{I}_{0}\left(\frac{\mathrm{A}-\mathrm{B}}{2} \mathrm{z}\right)
$$

Here $\mathrm{I}_{0}$ is the modified Bessel function of order zero, and $\mathrm{A}, \mathrm{B}$ are two constants to be calculated from the formulas

$$
\mathrm{A}+\mathrm{B}=\frac{2 \mathrm{~b} \beta-\mathrm{a} \gamma-\mathrm{c} \alpha}{\mathrm{ac}-\mathrm{b}^{2}} ; \quad \mathrm{AB}=\frac{\alpha \gamma-\beta^{2}}{\mathrm{ac}-\mathrm{b}^{2}} ; \mathrm{A}>\mathrm{B}
$$

Again, this probability density obviously satisfies the Stoka theorem, as it is a function of the joint invariants from equation (42). And so do the statistics of the variable Z, i.e. its mean and variance, for they can be calculated as

$$
\langle Z\rangle \equiv \frac{1}{2} \frac{A+B}{A B}=\frac{1}{2} \frac{2 b \beta-a \gamma-c \alpha}{\alpha \gamma-\beta^{2}} ; \quad \operatorname{var}(Z) \equiv \frac{1}{2} \frac{A^{2}+B^{2}}{A^{2} B^{2}}=\frac{1}{2}\left(\frac{2 b \beta-a \gamma-c \alpha}{\alpha \gamma-\beta^{2}}\right)^{2}-\frac{a c-b^{2}}{\alpha \gamma-\beta^{2}}
$$

We thus have the interesting conclusion that the essential statistics related to variable $\mathrm{Z}$ do not depend but on its coefficients and the values of the curvature parameters characterizing the point of contact.

\section{Contact Force by a Statistic}

First of all, the previous theory can help us secure, from a theoretical point of view, a purely statistical connotation in the curvature space itself. Assume indeed, that $\mathrm{a}, \mathrm{b}$ and $\mathrm{c}$ are some variations of the curvature parameters $\alpha, \beta$ and $\gamma$, respectively, over the ensemble of contact points representing the instantaneous surface of a nucleon. It thus turns out that the quantity with values given by equation (44), is actually a variation of the second fundamental form, controlled only by the variations of its coefficients. It is a statistical variable let us call $\mathrm{dZ}$ in order to show its differential nature - which has, according to equation (48), the expectation

$$
\langle\mathrm{dZ}\rangle \equiv \frac{1}{2} \frac{\mathrm{A}+\mathrm{B}}{\mathrm{AB}}=\frac{1}{2} \frac{2 \beta \mathrm{d} \beta-\gamma \mathrm{d} \alpha-\alpha \mathrm{d} \gamma}{\alpha \gamma-\beta^{2}}
$$

and the variance

$$
\left\langle[\Delta(\mathrm{dZ})]^{2}\right\rangle \equiv \frac{1}{2} \frac{\mathrm{A}^{2}+\mathrm{B}^{2}}{\mathrm{~A}^{2} \mathrm{~B}^{2}}=\frac{1}{2}\left(\frac{2 \beta \mathrm{d} \beta-\gamma \mathrm{d} \alpha-\alpha \mathrm{d} \gamma}{\alpha \gamma-\beta^{2}}\right)^{2}-\frac{\mathrm{d} \alpha \mathrm{d} \gamma-(\mathrm{d} \beta)^{2}}{\alpha \gamma-\beta^{2}}
$$

From these formulas we get a statistic having a special geometrical meaning:

$$
\left\langle[\Delta(\mathrm{dZ})]^{2}\right\rangle-\langle\mathrm{dZ}\rangle^{2} \equiv\left\langle\mathrm{d} \mathrm{Z}^{2}\right\rangle-2\langle\mathrm{dZ}\rangle^{2}=\frac{1}{4}\left(\frac{2 \beta \mathrm{d} \beta-\gamma \mathrm{d} \alpha-\alpha \mathrm{d} \gamma}{\alpha \gamma-\beta^{2}}\right)^{2}-\frac{\mathrm{d} \alpha \mathrm{d} \gamma-(\mathrm{d} \beta)^{2}}{\alpha \gamma-\beta^{2}}
$$

The right hand side of this formula carries indeed a special meaning: it is the Riemannian metric which can be built by the methods of absolute geometry for the space of the $2 \times 2$ matrices, having the curvature matrices with null Gaussian curvature as points of the absolute quadric (Mazilu, Agop, 2012). This is the Klein model of the so-called 'fourth geometry of Poincaré', in the modern views (see Duval, Guieu, 1998). In fact, one can prove that the quadratic form (50) is just the Cartan-Killing metric of a homographic action of the $2 \times 2$ real symmetric matrices. This is indeed the quadratic form 


$$
\frac{1}{4}\left(\omega_{2}^{2}-4 \omega_{1} \omega_{3}\right)
$$

where $\omega_{1,2,3}$ are the 1 -forms from equation (12).

Now, in order to introduce the contact force in our formalism, we only need to adopt the natural hypothesis that the deformation it induces is also to be accounted for by the variation of curvature. The considerations above just show a logical way toward that connection. Specifically, we leave the realm of infinitesimal deformation expressed by an auxiliary vector $|y\rangle$, and adopt its generalization through the apolarity condition (23). The vector representing the deformation, is then basically that from equation (27). Considering, for the sake of illustration, the small parameter $\varepsilon$, as well as a constant local stretch of the surface metric, included in the variation of the curvature parameters, the metric tensor expressing this deformation is given by equation (28) as

$$
\left(\begin{array}{cc}
1+\left(\mathrm{v}^{2}\right)^{2} & -\mathrm{v}^{1} \mathrm{v}^{2} \\
-\mathrm{v}^{1} \mathrm{v}^{2} & 1+\left(\mathrm{v}^{1}\right)^{2}
\end{array}\right)
$$

The eigenvalues of this matrix are 1 and $1+\langle v \mid v\rangle$. The corresponding eigenvectors are

$$
\left|\mathrm{e}_{1}\right\rangle=\left(\begin{array}{c}
\mathrm{v}^{1} \\
\mathrm{v}^{2}
\end{array}\right) ; \quad\left|\mathrm{e}_{2}\right\rangle=\left(\begin{array}{c}
-\mathrm{v}^{2} \\
\mathrm{v}^{1}
\end{array}\right)
$$

respectively. We choose their components as the convenient differential forms $\phi^{1,2}$ from the definition of contact force in equation (35). Accordingly the contact force can be described as a vector in the local tangent plane, whose components in the two orthogonal eigendirections of the metric tensor are given by

$$
|f\rangle=\left(\begin{array}{c}
d \omega_{1}^{3} \wedge v^{1}+d \omega_{2}^{3} \wedge v^{2} \\
-d \omega_{1}^{3} \wedge v^{2}+d \omega_{2}^{3} \wedge v^{1}
\end{array}\right)
$$

The first one of these components is null according to definition (35): naturally, there is no component of contact force along a direction, if there is no deformation along that direction. As to the second component, using equation (27), it is

$$
f_{2}=d \omega_{1}^{3} \wedge\left[\omega_{1} s^{1}+\left(\omega_{2} / 2\right) s^{2}\right]+d \omega_{2}^{3} \wedge\left[\left(\omega_{2} / 2\right) s^{1}+\omega_{3} s^{2}\right]
$$

Assuming now the gauge definition of the variation of curvature, as given by equations (10) and (11) this contact force becomes

$$
\begin{aligned}
& \mathrm{f}_{2}=\left[\left(\mathrm{d} \lambda-\omega_{2} / 2\right) \omega_{1}-\omega_{2} \omega_{3} / 2\right]\left(\omega_{1}^{3} \wedge \mathrm{s}^{1}\right)+\left[\left(\mathrm{d} \lambda+\omega_{2} / 2\right) \omega_{2} / 2+\omega_{1}{ }^{2}\right]\left(\omega_{2}^{3} \wedge s^{1}\right) \\
& \quad+\left[\left(\mathrm{d} \lambda-\omega_{2} / 2\right) \omega_{2} / 2-\omega_{3}{ }^{2}\right]\left(\omega_{1}^{3} \wedge \mathrm{s}^{2}\right)+\left[\left(\mathrm{d} \lambda+\omega_{2} / 2\right) \omega_{3}+\omega_{1} \omega_{2} / 2\right]\left(\omega_{2}^{3} \wedge s^{2}\right)
\end{aligned}
$$

Recall that we are using here the exterior multiplication in the tangent plane at a point of nucleon surface, not in the space of curvature parameters! With equations (7), (23) and (25) this 2-form is

$$
\mathrm{f}_{2}=\left\{\mathrm{d} \lambda\left[\left(\omega_{2} / 2\right)(\gamma-\alpha)+\beta\left(\omega_{1}-\omega_{3}\right)\right]+(\alpha+\gamma)\left[\left(\omega_{2} / 2\right)^{2}-\omega_{1} \omega_{3}\right]\right\}\left(\mathrm{s}^{1} \wedge \mathrm{s}^{2}\right)
$$

After some lengthy, but otherwise straightforward calculations, based on equation (12), we reduce this force to its final expression

$$
\mathrm{f}_{2}=(\alpha+\gamma)\left\{\mathrm{d} \lambda \mathrm{d} \xi+\left(\omega_{2} / 2\right)^{2}-\omega_{1} \omega_{3}\right\}\left(\mathrm{s}^{1} \wedge \mathrm{s}^{2}\right)
$$

where $\xi$ is the variable defined in equation (31), by the ratio of the two curvatures.

Just like the classical surface tension, this force contains the mean curvature of the surface in a point. However, unlike that classical expression it also depends on the variance of the increment of the second fundamental form due to a proces of 'wrinkling' at the contact, a phenomenon expressed by the variations of curvature parameters. If either one of the mean and Gaussian curvatures is not affected by this 'wrinkling', 
the magnitude of the contact force thus defined can be expressed exclusively by the Riemannian metric of the space of curvature parameters.

\section{Conclusions and Outlook}

A nucleon inside nucleus, can be conceived as a convex body limited in space by an irregular surface in permanent transformation due to interactions with the other nucleons. If it is to have some understanding of the strong and weak forces inside nuclear matter, then we have to describe a very first instance of the interactions between nucleons, namely the contact. There are two aspects of the geometrical and physical theory of contact: first the a priori choice of the location on the host surface of a generic nucleon, then the measure of the contact spot, due to the neighboring nucleons and their contact forces.

A natural idea about the physical description of the contact on the host surface, is that this contact is local, at least at a certain scale, and changes the curvatures of the surface at the location it takes place. This fact has the twofold consequence that can be clearly put into mathematical form: on one hand, a deformation of surface expressed by local curvature changes, on the other a certain definition of the variation of the curvature vector. These two mathematical results converge into a logical definition of the contact force as a differential 2-form that generalizes the classical definition of superficial tension.

The space of curvature parameters can be organized as a Riemann space, whose metric, has the precise statistical meaning as the variance of a certain deviation of the second fundamental form from its nominal value, due to the variation of its coefficients (the curvature parameters). The contact force, as defined here, is proportional with the nominal mean curvature as in the classical case, but also involves the variance of the second fundamental form, therefore the Riemannian metric of the space of the curvature parameters. This fact can have important consequences in the description of the dynamics of a nucleon inside nucleus by a gauge theory. But unlike the classical case of Shapere and Wilczek, the gauge is defined by the variation of local curvature, and asks for a proper definition of the instantaneous nucleon surface. This one can be conceived as an ensemble of 'elements of contact', whose characteristic contact force already contains a statistical element through the variance of the second fundamental form.

The actual (spatial) instantaneous surface of a nucleon is nevertheless a matter of further elaboration. However, one could say that this elaboration can take advantage of a sound guidance, both from the classical Fresnel theory of the wave surface of light, and the modern theory of a holographic universe, according to which the interior of a nucleon should be structured as a hologram ('t Hooft, 1993; Susskind, 1994). In this respect, the actual surface of the nucleon can even be taken as a fuzzy sphere in the sense of Madore (Madore, 1992). This can be quite a natural approach, in view of the fact that with the variation of the curvature parameters, as presented in this work, we are actually in the realm of $\mathfrak{s l}(2, \mathrm{R})$ Lie algebra.

\section{References}

Berry, M. V. (1985): Classical Adiabatic Angles and Quantal Adiabatic Phase, Journal of Physics A: Mathematical and General, Vol. 18, pp. 15 - 27 (Reprinted in the collection Shapere, Wilczek, 1989c)

Błocki, J., Świątecki, W. J. (1981): A Generalization of the Proximity Force Theorem, Annals of Physics, Vol. 132, pp. 53-65

Bloore, F. J. (1977): The Shape of Pebbles, Mathematical Geology, Vol. 9, pp. 113-122

Burnside, W. S., Panton, A. W. (1960): The Theory of Equations, Dover Publications

Cartan, E. (2001): Riemannian Geometry in an Orthogonal Frame, World Scientific Publishing, Singapore

Domokos, G., Gibbons, G. W. (2013): Geometrical and Physical Models of Abrasion, arXiv:1307.5633v1

[physics.geo-ph] 
Duval, C., Guieu, L. (1998): The Virasoro Group and the Fourth Geometry of Poincaré, arXiv: mathDG/9806135v1

Firey, W. J. (1974): Shapes of Worn Stones, Mathematika, Vol. 21, pp. 1 - 11

Gradshteyn, I. S., Ryzhik, I. M. (2007): Table of Integrals, Series and Products, Seventh Edition, A. Jeffrey \& D. Zwillinger Editors, Academic Press-Elsevier, Inc.

Guggenheimer, H. W. (1977): Differential Geometry, Dover Publications, New York

Hannay, J. H. (1985): Angle Variable Holonomy in Adiabatic Excursion of an Integrable Hamiltonian, Journal of Physics A: Mathematical and General Vol. 18, pp. 221-230 (Reprinted in the collection Shapere, Wilczek, 1989c)

Lowe, P. G. (1980): A Note on Surface Geometry with Special Reference to Twist, Mathematical Proceedings of the Cambridge Philosophical Society, Vol. 87, pp. $481-487$

Madore, J. (1992): The Fuzzy Sphere, Classical and Quantum Gravity, Vol. 9, pp. 69-80

Mazilu, N. (2004): The Stoka Theorem, a Side Story of Physics in Gravitation Field, Supplemento ai Rendiconti del Circolo Matematico di Palermo, Vol. 77, pp. 415-440

Mazilu, N., Agop, M. (2012): Skyrmions - a Great Finishing Touch to Classical Newtonian Philosophy, Nova Publishers, New York

Mazilu, N., Ioannou, P. D., Diakonos, F. K., Maintas, X. N., Agop, M (2013): A Quark-Independent Description of Confinement, Modern Physics Letters, Vol. A28, 1350126

Mazilu, N., Ioannou, P. D., Agop, M (2014): The Classical Theory of Light Colors: a Paradigm for Description of Particle Interactions, Bulletin of the Polytehnic Institute of Iaşi, Vol. 60, (to appear)

Pommaret, J.- F. (2013): The Mathematical Foundations of Gauge Theory Revisited, arXiv:1310.4686v1 [math.AP]

Shapere, A., Wilczek, F. (1989a): Geometry of Self-Propulsion at Low Reynolds Number, Journal of Fluid Mechanics, Vol. 198, pp. 557 - 585 (Reprinted in the collection Shapere, Wilczek, 1989c)

Shapere, A., Wilczek, F. (1989b): Gauge Kinematics of Deformable Bodies, American Journal of Physics, Vol. 57, pp. 514 - 518 (Reprinted in the collection Shapere, Wilczek, 1989c)

Shapere, A., Wilczek, F. (1989c): Geometric Phases in Physics, World Scientific Publishing Co., Singapore Spivak, M. (1999): A Comprehensive Introduction to Differential Geometry, Publish or Perish, Inc., Houston, Texas

Stoka, M. I. (1968): Géométrie Intégrale, Mémorial des Sciences Mathématiques, Gauthier-Villars, Paris Susskind, L. (1994): The World as a Hologram, arxiv: hep-th/9409089

't Hooft, G. (1993): Dimensional Reduction in Quantum Gravity, arxiv: gr-qc/9310026 